\title{
Thank you letter for previous issue on aerospace medicine
}

\author{
Mark Lipset
}

As a McGill University alumni and a participant in the Canadian Space Agency's Aerospace Medicine Elective and the International Space University Space Studies Program, I would like to thank you for your engaging issue on aerospace medicine (Issue 13.2).

The aerospace medicine issue of the MJM brought to light how fortunate we are as Canadians to live in a country in which the fruition of our dreams are supported though entities such as the Canadian Space Agency. The Canadian Space Agency has been a driving force in Canadian aerospace development and education for decades. Dr. David Saint-Jacques' and Dr. Robert Thirsk's articles touched on the similarities between astronaut and physician training; similarities that are the foundation of the Royal College of Physicians and Surgeons of Canada's (RCPSC) physician development CanMEDS roles (which underscore the roles of being a medical expert, communicator, collaborator, manager, health advocate, scholar, and professional). These roles provide the fundamental backbone to becoming an excellent professional and providing the highest quality service.

Through their numerous research initiatives, the Canadian Space Agency has provided opportunities for physicians to enhance these CanMEDS roles to an even greater level, as illustrated by Jason Clement. This synergistic relationship between space and medical education is an enviable construct that we as Canadian medical students and residents should take full advantage of. In light of this, I would propose that the RCPSC, individual medical schools, and the

*To whom correspondence should be addressed: Mark Lipset McGill University, Faculty of Medicine Montreal, Quebec, Canada, H3G 1A4

Tel: (514) 771-8890

Email: mlipsett@gmail.com
Canadian Government at large recognize this synergistic relationship and capitalize on it in order to provide a greater opportunity to enhance the well-rounded education of Canadian physicians so that they may provide the best level of patient care.

The financial investment of tax payer's dollars to the Canadian Space Agency to support research and education in aerospace medicine not only provides unique learning experiences for passionate and accomplished young professionals and students, but also provides a "double-return" on investment by enhancing both Canada's space program and facets of healthcare delivery. Thus, this continued financial investment in aerospace medicine and subsequent creation of learning and growth opportunities is imperative to the further growth of future aerospace leaders and health professionals. 